\title{
Optimum Design of Passive Power Filter (PPF) at the output of 5-level CHB-MLI using Genetic
}

\section{Algorithm (GA)}

\author{
BasemAlamri \\ Taif University (TU) \\ Taif, Saudi Arabia \\ B.alamri@tu.edu.sa
}

\author{
Christos Marouchos \\ Cyprus University of Technology \\ Cyprus \\ Christos.marouchos@,cut.ac.cy
}

\author{
Mohamed Darwish \\ CEDPS, Brunel University \\ London, UK \\ Mohamed.Darwish@brunel.ac.uk
}

\begin{abstract}
While harmonics have adverse effects on both power utilities and customers, harmonic filtering is considered the most widely applied method among different harmonicmitigation techniques. Passive power filters (PPFs) are currently more economical and commonly applied than active power filters (APFs). The problem of passive power filter (PPF) design is considered to be a combinatorial optimisation problem that can be solved by applying artificial intelligence. For PPF design, heuristic methods are powerful optimisation techniques and have many advantages such as: no requirement for detailed information about the power system and ability to achieve optimum PPF design compared to the conventional method. In addition, the cost of PPF implementation can be added to the optimisation objective, which is not considered in conventional design. The Authors of this paper propose an optimisation model based on genetic algorithm (GA) to design a composite PPF. As a case study, the model is applied to find the optimum filter design at the output of 5-level cascaded H-bridge multilevel invert (CHB-MLI). MATLAB-SIMULINK is used for the modelling and simulation.
\end{abstract}

Index Terms - Cascaded H-Bridge Multilevel Inverter (CHBMLI), Passive Power Filters (PPF), Genetic Algorithm (GA), Harmonics

\section{INTRODUCTION}

Power system harmonics have adverse effect on power systems and equipment such as overloading, heating, reduction of life, aging of insulators and many other effects. Power harmonics is considered a major issue of power quality as it affects both sides the utility and the customer. Over the last 30 years this phenomena has been studied extensively. In practice, there are many sources which generate harmonics in power systems. Non-linear loads are known to be the major source of harmonics in power systems. With the huge developments in power electronics technology, there was a rapid growth of non-linear loads in electrical power systems. Among non-linear loads, power electronics converters, and due to the use of power semiconductor switching in its operation, are of the main sources of harmonics in modern electrical power systems. With the need for more penetration of renewable energy sources such as solar PVs and wind turbines, the future power systems will rely more in power conversion technologies which result in more harmonics generation within the system. Electrical power system operators should plan the operation of the system to mitigate the generated harmonics efficiently in order to meet the harmonics levels recommended by international power quality standards such as IEEE-519.

In practice, there are many applied techniques to eliminate harmonics. Among these techniques, harmonic filtering is very widely applied. Power harmonic filters can be classified mainly into: 1) passive power filters (PPF), and 2) active power filters (APF) [1] . Nowadays, PPFs are very commonly used in power systems due to its many advantages compared to APFs such as: low cost, almost maintenance free, simple structure, and simple operation. This paper investigates the application of PPFs for harmonic mitigation.

The survey of published papers on power electronic filtering using PPFs shows a great deal of interest over the past view years. The applied methods in PPF design can be classified into two main approaches: 1) conventional approach, and 2) heuristic approach. The first approach, rely more on engineering experience, needs a lot of date about the system and usually does not achieve optimum design. On the other hand, heuristic methods are very powerful techniques and can be implemented to design PPFs. The heuristic approach optimizes the design of PPF. Different objectives can be minimized such as THD, filter cost subject to operational and technical constraints. Heuristic design of PPFs does not need much information of the system and result in optimum design of filter which has better harmonic suppression and lower investment cost compared to conventional design.

Genetic Algorithm is one of the most powerful heuristic optimization techniques and it has many advantages compared to other optimization techniques [2]. This paper proposes Genetic Algorithm (GA) to design PPF connected at the output of 5-level cascaded H-Bridge multilevel inverter (CHB-MLI) as a case study. Selective Harmonic Elimination (SHE) is applied to control the 5-level CHB-MLI as it is very widely applied technique. MATLAB-Simulink is to be used as a simulation environment. The expected result of this paper is to find the optimum design of the output PPF which result in minimizing both THD and filter cost. 
II. (CHB-MLI)

Multilevel voltage source inverters are classified into three basic topologies which are namely: 1) Neutral-Point Clamped Multilevel Inverters (NPC-MLI) , 2) Fly Capacitor Multilevel Inverters (FC-MLI), and 3) Cascaded H-Bridge Multilevel Inverters (CH-MLI).

The NPC-MLI and FC-MLI are widely used for industrial medium voltage- high power applications when just low number of levels (typically three) is required. On the other hand, the CHB-MLI is most suitable for high voltage-high power, HVDC and utility applications. Main reason is that the CHB-MLI has modular structure which can be extended for high number of levels with no much complexity. Furthermore, with CHB-MLI, higher power and voltage capability can be achieved at lowest number of required devices compared to NPC-MLI and FC-MLI.

The CHB-MLI uses series connection of single phase Hbridge inverters with separate dc sources. The operating principle is that each bridge (cell) will generate three different voltages and the voltage output waveform can be synthesized by the sum of the voltages generated from each cell. The separate dc sources might be solar panel PV cells or fuel cells. [3]

In this paper, the inverter is designed to be connected to 11 $\mathrm{kV}$ line to line grid voltage. The switching devices have been selected to be of IGBT type CM400HB-90 H, which has a blocking voltage capability of $4.5 \mathrm{kV}$, and a maximum forward current of $400 \mathrm{~A}$. Based on the required output voltage and the selected IGBT blocking voltage, the inverter is designed as 5-level CHB-MLI as shown in Fig. 1. Each cell is connected to a dc link supply of $4.1 \mathrm{kV}$. Modulation index of (0.9) was used throughout the analysis. The inverter was modelled in MALTAL-SIMULINK with main objective of finding the optimum design for the output PPF.

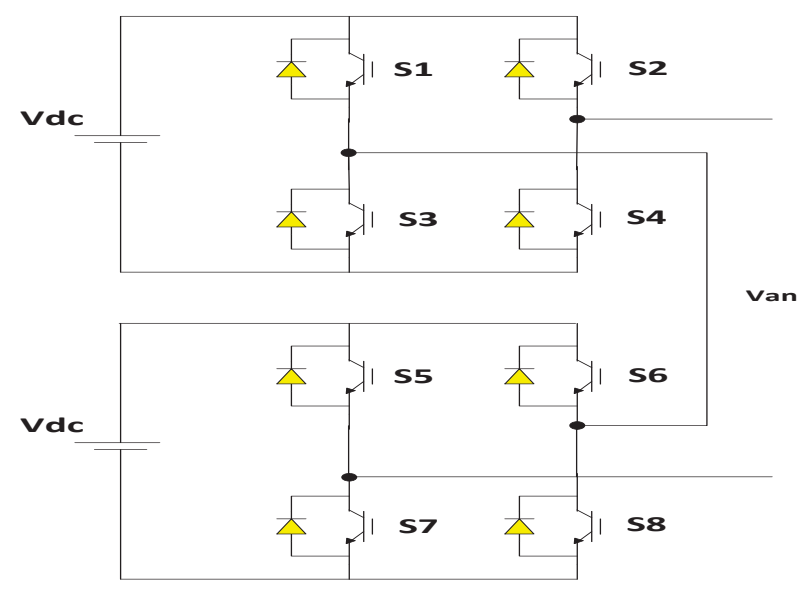

Fig. 1. Single-phase 5-level cascaded H-bridge inverter circuit layout.

\section{PASSIVE POWER FILTER (PPF) OPTIMIZATION}

Power harmonics have adverse effects on both power utilities and customers. In practice, harmonic filtering is considered the most widely applied method among different harmonicmitigation techniques. The function of the added shunt filter is to ensure that the harmonics profile is well below the recommended limits, as per the IEEE-519 power-quality standard. The proposed PPF should be based on cost-effective design.

PPFs are widely employed in electrical and industrial power systems compared to APFs because PPFs are simple in design, cheap and effective. There are different PPF topologies, which are classified into tuned and high-pass filters. Among all PPF topologies, the composite topology is commonly applied for high-power applications to assure reduction of harmonics below IEEE-519 limits. A typical circuit layout for the implemented composite PPF at the output of the CHB-MLI is demonstrated in Fig. 2.

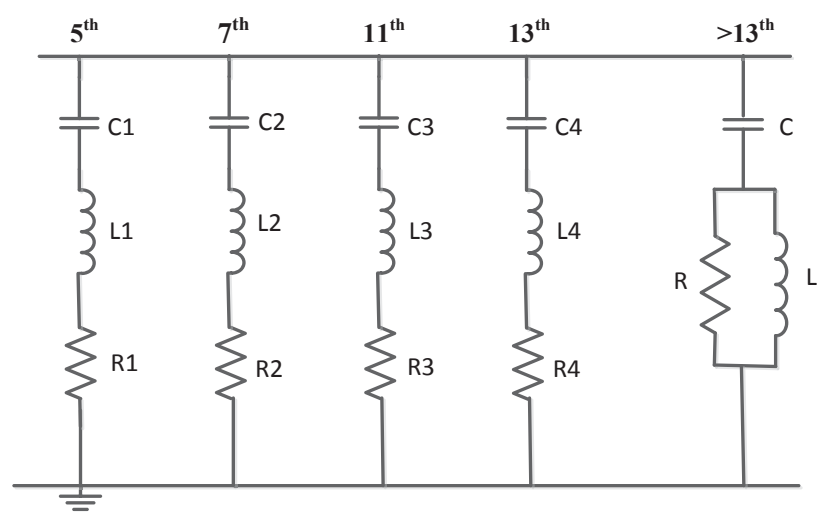

Fig. 2. Composite PPF circuit layout.

In such filter topology, the shunt single-tuned filters are to be tuned to eliminate low-order harmonics and the second-order high-pass filter eliminates most of the higher-frequency harmonics. The PPF provides a low-impedance path for the tuned-harmonics currents and provides reactive-power compensation at the fundamental frequency [5].

The optimum design of PPF for electrical power systems should reduce the THD of currents and voltage at PCC below the recommended limits stated in power-quality standards. Furthermore, appropriate PPF design should minimize proposed filter costs. In this study, an optimisation model of PPF design based on genetic algorithm (GA) is proposed as a heuristic approach has many advantages over the conventional design approach.

PPF design is a complicated task as it should consider many parameters in the design stage such as: filter size, filter cost, power losses, system configuration and the harmonics limits 
defined in power-quality standards. The following are the key design principles and considerations for appropriate PPF design $[6,7]$.

$>$ Filter-design parameters C, L and R should satisfy the filtering objective.

$>$ The filter should compensate the system reactive-power demand at fundamental frequency.

$>$ Lower harmonic contents of voltage and current at PCC below the limits recommended in power-quality standards.

$>$ Minimize total filter cost

$>$ Higher power factor at PCC.

$>$ Avoid series or parallel resonance between filter impedance and system impedance.

\section{GENETIC ALGORITHM (GA)}

GA is a heuristic global evolutionary optimization algorithm which works based on mechanics of natural selection and genetics. GA is a simple and easy to implement, hence, it can be easily applied to solve for optimum design of shunt passive power filters (PPF). In any GA optimization, The main process consists of five steps which are: 1) Initialization of the population, 2) Evaluation of fitness function, 3) Selection, 4) Apply genetic operators, and 5) Stopping Criterion. [8]

\section{STEP_1: Initialization}

The algorithm is initialized. The parameters of the optimization problem are coded either in a binary or floatingpoint string. Based on the coded parameters, a set of solutions is randomly generated.

\section{STEP_2: Evaluation of Fitness Function}

In order to test the goodness of a generated solution, a fitness function is to be applied as an evaluation tool. In this analysis, an objective function was defined as a fitness value (FV). It is of great importance to define the fitness function critically and very carefully as it has a great effect on the quality of the optimized solution. The objective function should satisfy the optimization objectives.

\section{STEP_3: Selection}

In this step, parents are chosen based on selection rules in order to produce offspring chromosomes. The selected parents are the main contributors of the next generation. The fittest individual are likely to survive and the less fit are to be eliminated.

\section{STEP 4: Crossover and mutation}

In crossover a number of bits are swapped between parents. Here, some genes are exchanged to form a new improved combination. Another very important operator to be applied is called Mutation, in which the genes are alerted.

\section{STEP_5: Stopping Criterion}

This step is very important as it tells the algorithm when to stop and terminate.

\section{GA-BASED OPTIMIZATION FOR PPF DESIGN}

This section proposes an optimized PPF design approach based on GA. The three optimisation objectives are: 1) THD, 2) system cost, and 3) reactive power compensation. The filter to be optimized comprises multiple single-tuned filters in parallel with a $2^{\text {nd }}$ order high-pass filter. The unknown variables are the fundamental reactive-power $Q_{c 1}$ injected by each filter branch. Once determined, the filter parameters can be easily calculated. The model is for three phase system.

\section{A. Single-Tuned PPF Design}

In the design of shunt single-tuned PPF tuned for the $n^{\text {th }}$ harmonic order, the branch impedance $Z_{S H n}$ can be expressed as follows [9]:

$Z_{S H n}=R_{S H}+j\left(n X_{L S H}-\frac{X_{C S H}}{n}\right)$

First, the reactive power, $Q_{S H 1}$ supplied by the shunt singletuned branch at fundamental frequency is determined. Based on the value of $Q_{S H 1}$, the magnitude of the fundamental capacitive reactance of the shunt-tuned branch $X_{C S H}$ can be calculated simply by applying the following formula:

$X_{C S H}=3 \times \frac{n^{2}}{n^{2}-1}\left(\frac{V_{L 1}^{2}}{Q_{C 1}}\right)$

Where $V_{L 1}$, is the fundamental voltage at the tuned shunt branch. In practice, the shunt-capacitor components are available in discrete incremental steps of reactive-power ratings. An incremental discrete step of $50 \mathrm{kVar}$ was used as per recommendations for low-voltage industrial power application $[9,10]$.

Then, the magnitude of the fundamental inductive reactance $X_{C S H}$ and the resistance $R_{S H}$ of the shunt single-tuned branch is calculated:

$X_{L S H}=\frac{X_{C S H}}{n^{2}}$

$R_{S H}=\frac{X_{C S H}}{n Q}$

In this study, the optimum value of the filter quality factor $(Q)$, was considered to be 60 for all single-tuned filters to assure the best filter performance $[60,94]$. 


\section{B. High-Pass PPF Design}

Similarly, for the high-pass filter branch, the impedance of the high-pass filter is given by [6]:

$Z_{H n}=-j \frac{X_{C H}}{n}+\left(\frac{1}{R_{H}}+\frac{1}{j n X_{L H}}\right)$

Once the injected fundamental reactive power $Q_{c 1}$ of the high-pass filter is determined, the magnitude of the fundamental capacitive reactance of the shunt high-pass filter branch $X_{C H}$ can be calculated simply by applying the following formula:

$X_{C H}=3 \times \frac{n^{2}}{n^{2}-1}\left(\frac{V_{L 1}^{2}}{Q_{C 1}}\right)$

Then, the magnitude of the resistance $R_{H}$, the fundamental inductive reactance $X_{L H}$ and the shunt high-pass filter branch is calculated:

$R_{H}=\frac{X_{C H}}{n}$

$X_{L H}=\frac{m R_{H}}{n}$

Here, $m$ is the damping-time constant ration, which usually takes a value between $[0.5,2]$. In this study, $m$ is chosen to be equal to (0.5) as this results in minimum losses [11].

\section{Objective Function and Constraints Formulation}

In order to satisfy the key design principles and considerations given in Table 4-6, the following objectives have been set for the optimisation:

Objective 1: Minimize the total harmonic distortion of the output voltage $\mathrm{THD}_{\mathrm{v}}$

$f_{1}=\operatorname{Min} \cdot T H D_{v}$

$T H D_{v}=\sqrt{\sum_{n=2}^{\infty}\left(\frac{U_{n}}{U_{1}}\right)^{2}}$

Where:

$\mathrm{THD}_{\mathrm{v}}$ is the total harmonic distortion of output voltage.

$\mathrm{U}_{\mathrm{n}}$ is the $\mathrm{n}^{\text {th }}$ harmonic of output voltage.

$\mathrm{U}_{1}$ is the fundamental of output voltage.
Objective 2: Minimize the system-capital cost and energyloss cost.

$$
f_{2}=\operatorname{Min} \cdot\left(f_{\text {Capital_Cost }}+f_{\text {Operating_Cost }}\right)
$$

Richards et al. presented a method to compute the total capital-investment cost of a single-tuned PPF [13]. Here, the capital-investment cost of the filter is calculated based on the size MVAR ratings of the filter. In this study, the cost of a single-tuned and high-pass filter is calculated as follows:

$f_{\text {Filter_Cost }}(£)=U_{C} \times Q_{C}+U_{L} \times Q_{L}$

Where, $U_{C}$ and $U_{L}$ are the unit cost of capacitor and inductor in (£/MVARs), respectively. In this optimisation, both cost of capacitor and inductor are assumed to be equal in order to maintain simplicity and obtain generic optimisation, so that $U_{C}=U_{L}=10,000 £ / \operatorname{MVAR}[14]$.

The operating cost of the system is mainly the cost of energy losses. It is calculated in terms of equivalent annualized capital cost by applying the present value factor as shown below [96]:

$f_{\text {Operating_Cost }}(£)$

$$
\begin{aligned}
& =H \times U_{\text {Energy }} \times \frac{(1+i)^{y}-1}{i(1+i)^{y}} \\
& \times P_{\text {loss }}
\end{aligned}
$$

Where:

$H:$ is total operating hours annually (h/year)

$U_{\text {Energy }}$ is the energy cost in $(\mathfrak{f} / \mathrm{kWh})$,

$i:$ is the annual interest rate for capital cost, which is assumed to be $5 \%$,

$y:$ is the levelaization period or system lifetime (years), which is assumed to be 15 years.

Objective 3: Maximize the generated fundamental reactive power supplied by the filter. This will give the maximum possible power factor for the optimisation.

$f_{3}=\operatorname{Max} . \sum Q_{i}$

Where $Q_{i}$ is fundamental reactive power supplied by the $i^{\text {th }}$ filter.

In order to combine all three objectives into one function and simplify the fitness function, a weighted multi-objective 
technique is implemented. The three objectives transformed into one objective function as shown below:

$$
\text { Min. }\left[w_{1} f_{1}+w_{2} f_{2}+w_{3}\left(C-f_{3}\right)\right]
$$

Here, $\mathrm{C}$ is a large constant to ensure that $\left(C-f_{3}\right)$, is always a positive number. In this equation, the weighting factors $\mathrm{w}_{1}$, $\mathrm{w}_{2}$ and $\mathrm{w}_{3}$ are given the values $0.5,0.4$ and 0.1 , respectively.

GA will minimize the objective function stated previously. However, there are some constraints for the optimisation that should be considered, as follows:

\section{IEEE-519 Standard}

Based on the standard, for voltage levels up to $69 \mathrm{kV}$, $\mathrm{THD}_{\mathrm{v}}$ should be kept under 5\%, and non-individual voltage harmonics should exceed $3 \%$.

\section{Load Power Factor}

PPF compensation for reactive power should maximize the power factor, and at the same time, the system should not be overcompensated.

$Q_{\min } \leq \sum Q_{i} \leq Q_{\max }$

\section{SIMULATION RESULTS AND DISCUSSION}

The proposed optimisation model was applied to solve for the optimum deign of PPF at the output of 5-level CHB-MLI. The inverter is connected to grid at $11 \mathrm{kV}, 5 \mathrm{MW}$ and 0.8 lagging power factor. The problem of SHE was obtained applying GA as explained in author's paper [15] assuming a modulation index of 0.9 . The optimum angles were found be

$\theta_{1}=9^{\circ}$ and $\theta_{2}=26.8^{\circ}$. The output three phase voltage waveform is shown in Fig. 3 . Here, the \%THD was found to be almost $9 \%$.

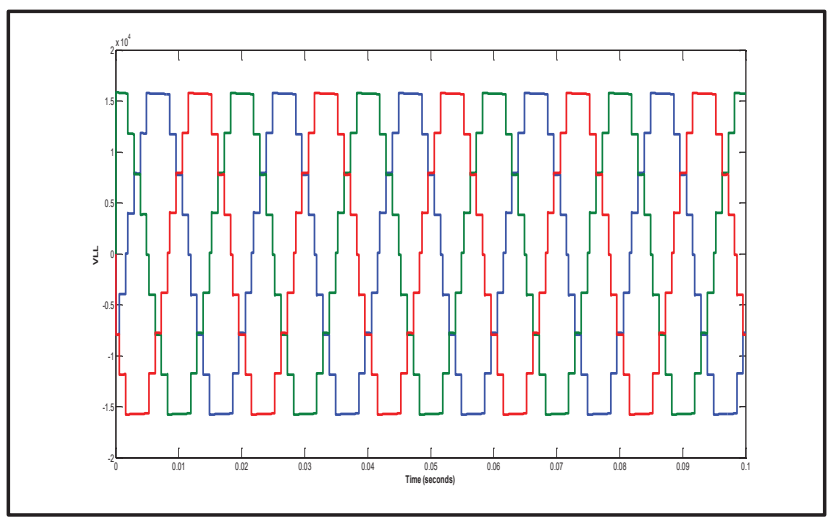

Fig. 3. Output three-phase voltage for 5-level CHB-MLI before filter implementation.
For the considered case study, the optimum composite passive power filter is designed to be with three single tuned shunt filters and one high pass filter as explained in Table 1. The optimum PPF size was 3.95 MVar whiłh is required to be added at the output of the 5-level CHB-MLI. As a result of implementing the composite PPF, the \%THD has been reduced to $4.9 \%$ which is below the specified IEEE-519 recommended harmonic limits. The three single tuned filters are tuned at the $7^{\text {th }}, 11^{\text {th }}$ and $13^{\text {th }}$ harmonics. The high pass filter is designed for the harmonics order beyond the $17^{\text {th }}$.

The three phase output voltage waveform after implementing the filter is presented in Fig. 4. It is clear that the quality of the output voltage waveforms were improved significantly.

TABLE I: Optimum PPF design based on GA

\begin{tabular}{lcccc}
\hline \multicolumn{5}{c}{ Proposed Passive Power Filter (PPF) } \\
\hline Filter Type & ST & ST & ST & HP \\
\hline Order & $7^{\text {th }}$ & $11^{\text {th }}$ & $13^{\text {th }}$ & $>17^{\text {th }}$ \\
Size $(\mathrm{kVar})$ & 450 & 600 & 450 & 2,450 \\
$\mathrm{C}(\mu \mathrm{F})$ & 3.94 & 5.26 & 3.94 & 21.5 \\
$\mathrm{~L}(\mathrm{mH})$ & 52.4 & 15.9 & 15.2 & 1.63 \\
$\mathrm{R}(\Omega)$ & 1.92 & 0.92 & 1.03 & 8.7 \\
Quality Factor & 60 & 60 & 60 & 5 \\
Total Filter Size $(\mathrm{kVar})$ & & 3,950 & \\
THD Before Filter $(\%)$ & & 8.99 & \\
THD After Filter $(\%)$ & & 4.90 & \\
\hline
\end{tabular}

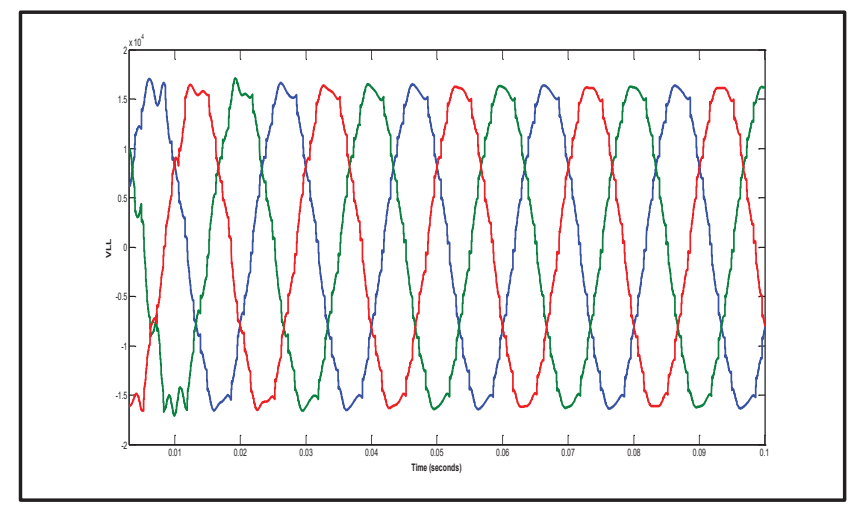

Fig. 4. Output three-phase voltage for 5-level CHB-MLI after filter implementation. 


\section{CONCLUSION}

Harmonics have adverse effects on both utility and customers. Different solutions were applied in practice to eliminate harmonics. However, passive Power Filters (PPF) are considered the most economic and widely applied method for harmonic mitigation in power systems. The aim of this paper was to propose and optimum design methodology based on GA for a composite PPF at the output of 5-levels CHB-MLI. The implemented PPF should improve the lagging power factor to between $(0.92$ and 0.98 ) and reduce the \% $\%$ THD at the output of the inverter. In addition, the total cost of the system, including filter cost and energy loss should be minimised. Such compensation cases require the implemented PPF to inject a total reactive power between 1.6 and 3.7 MVAR. The implemented model was applied successfully for the design of the PPF in which the \%THD was reduced to less than $5 \%$ and the quality of the output voltage waveform was improved significantly.

The conventional approach for PPF design, rely more on engineering experience, needs a lot of date about the system and usually does not achieve optimum design. However, heuristic approach optimizes the design of PPF. Different objectives can be minimized such as THD, filter cost subject to operational and technical constraints. Heuristic design of PPFs does not need much information of the system and result in optimum design of filter which has better harmonic suppression and lower investment cost compared to conventional design. The authors recommend the application of heuristic approach as explained in the proposed model in this paper. However, more cautions should be considered when defining the objective function as it affects the optimisation output critically.

\section{REFERENCES}

[1] Kazem, Hussein A. "Harmonic mitigation techniques applied to power distribution networks." Advances in power electronics, (2013)

[2] Verma, Vishal, and Bhim Singh. "Genetic-Algorithm-based design of passive filters for offshore applications." Industry Applications, IEEE Transactions on 46.4 (2010): 1295-1303.

[3] N. Mittal, B. Singh, S.P. Singh, R. Dixit and D. Komar, "Multi-Level Inverter: a Literature Survey on Topologies and Control Strategies", ICPCES, $2^{\text {nd }}$ International Conference on Power, Control and Embedded Systems, 2012.

[4] M.G.H. Aghdam, S.S. Fathi and A. Ghasemi, "The analysis of conduction and switching losses in three-phase OHSW multilevel inverter using switching functions", IEE PEDS 2005, Vol. 1, pp. 209218, 2005.

[5] A. Dastfan, H. Yassami and M. R. Rafiei, "Optimum design of passive harmonic filter by using game theory concepts," Intelligence Systems in Electrical Engineering Journal 4.4 pp.13-22, 2014.

[6] Z. Juan, G. Yi-nan and Z. Shu-ying, "Optimal design of passive power filters of an asymmetrical system based on genetic algorithm," Procedia Earth and Planetary Science, vol. 1, pp. 14401447, 2009.

[7] L. Huang, N. He and D. Xu, "Optimal design for passive power filters in hybrid power filter based on particle swarm optimization," in
Automation and Logistics, 2007 IEEE International Conference On, 2007, pp. 1468-1472.

[8] B. Alamri and M. Darwish, "Power loss investigation in HVDC for cascaded H-bridge multilevel inverters (CHB-MLI)," in PowerTech, 2015 IEEE Eindhoven, 2015, pp. 1-7.

[9] A. F. Zobaa and S. H. E. A. Aleem, "A New Approach for Harmonic Distortion Minimization in Power Systems Supplying Nonlinear Loads," Industrial Informatics, IEEE Transactions On, vol. 10, pp. 1401-1412, 2014

[10] "IEEE Standard for Shunt Power Capacitors," IEEE Std 18-2002 (Revision of IEEE Std 18-1992), pp. 1-20, 2002.

[11] Z. Juan, G. Yi-nan and Z. Shu-ying, "Optimal design of passive power filters of an asymmetrical system based on genetic algorithm," Procedia Earth and Planetary Science, vol. 1, pp. 1440 1447, 2009.

[12] Y. Cho and H. Cha, "Single-tuned passive harmonic filter design considering variances of tuning and quality factor," Journal of International Council on Electrical Engineering, vol. 1, pp. 7-13, 2011 .

[13] G. G. Richards, O. T. Tan, P. Klinkhachorn and N. I. Santoso, "Costconstrained power factor optimization with source harmonics using LC compensators," Industrial Electronics, IEEE Transactions On, pp. 266-270, 1987.

[14] M. Elmathana, A. Zobaa and S. Aleem, "Economical design of multiple-arm passive harmonic filters for an industrial firm-case study," in Harmonics and Quality of Power (ICHQP), 2012 IEEE 15th International Conference On, 2012, pp. 438-444.

[15] B. Alamri and M. Darwish, "Power loss investigation in HVDC for cascaded H-bridge multilevel inverters (CHB-MLI)," in PowerTech, 2015 IEEE Eindhoven, 2015, pp. 1-7. 